\title{
Document of diabetes care for residential and nursing homes
}

\author{
AJ Sinclair, CJ Turnbull, SCM Croxson
}

In the UK, there has been a rapid expansion in residential and nursing home care for elderly people with a corresponding reduction in long-term hospital care. Many of the residents are disabled and a large percentage are likely to have diabetes, although accurate information is not available. The needs of patients with diabetes living within institutions may be overlooked if follow-up strategies have been terminated, if a structured care plan is absent or if no 'nominated' carer is available, or if care staff have little knowledge of basic diabetes management. In addition, in smaller institutions, the number of residents with diabetes may be relatively small and care staff accordingly have little opportunity for gaining experience in diabetes care.

\section{Management problems}

Common problems making diabetes management more complex in these patients include:

- Irregular oral intake due to confusion and/or a poor appetite due to concurrent illness; dysphagia due to stroke or other neurological or gastroenterological disorders. Lack of regular calorific intake can be particularly troublesome for insulin-treated patients.

- Recurrent urine, chest and other infections rendering the diabetic person liable to hyperglycaemia and possibly ketosis with poor metabolic control.

- Leg ulcers and pressure sores which may rapidly deteriorate in diabetic patients.

- Dysphasia, dysarthria, or deafness and blindness which may make it difficult to communicate: needs are thus unrecognised and unmet.

- Increased vulnerability to hypoglycaemia (especially in those taking sulphonylureas or insulin), due to poor appetite, difficulties in ensuring sufficient and regular calorific intake, lack of glucose monitoring (urine or blood), lack of knowledge of symptoms and signs of hypoglycaemia which may be attenuated in older people with diabetes, polypharmacy (multiple drug therapy), and recurrent acute illness.

- Multiple pathology (especially cardiac and renal failure), increasing the likelihood of adverse drug reactions to prescribed medications. This may be exacerbated by infrequent review of existing medications.

- Inadequate facilities within institutions to cater for the dietary needs of residents with diabetes, and inadequate knowledge of catering staff on what and how to provide appropriate and varied food for diabetic residents, including the necessity of provision of snacks outside main meals.

Department of Geriatric Medicine and Gerontology, University of Birmingham, The Hayward Building, Selly Oak Hospital, Birmingham B29 6JD, UK

AJ Sinclair

Arrowe Park Hospital, Upton, Wirral, Merseyside L49 5PE, UK

CJ Turnbull

Bristol General Hospital, Bristol BD1 6SY, UK

SCM Croxson

This document was compiled by Professor AJ Sinclair and Dr CJ Turnbull from contributions from the following members of the Special Interest Group in Diabetes: Dr SJ Benbow, Dr B Kaufman, Dr JM Potter, Dr TJ Hendra, Dr R Gadsby

Accepted 25 September 1996

- Lack of adequate arrangements for regular diabetes review particularly for those discharged from hospital clinics and who are unable to attend general practitioners' surgeries.

- Lack of experience and training of institutional staff in diabetes care, which currently does not allow glucose monitoring to be undertaken and which prevents useful assistance with insulin administration.

- Insufficient provision of health professional input, particularly specialist nurses, dietitians, dental surgeons, opticians, state-registered chiropodists, and doctors, including hospital specialists. Where appropriate, financial assistance should be sought for those residents who require regular chiropody and have to pay extra for it.

\section{Improving care}

Patients in institutionalised settings have special needs and the following suggestions relate to how these needs can be met by improving care:

- Each resident should have an individual diabetes care plan agreed between patient (or relative), doctor (who is responsible for diabetic care) and homecare staff. This should include details relating to food intake, treatment, 
monitoring, glycaemic targets, personal care and observations, eg, weight, recording fluid intake where appropriate, etc.

- Increased community support from experienced health professionals such as diabetes specialist nurses and dietitians. Easy access to patients and educational advice given to care staff are essential.

- Diabetic patients living in residential nursing homes should have ready access to other specialist health professionals. In particular, diabetes-directed chiropody, orthotics and specially trained opticians. Arrangements should be made for appropriate referral to specialist hospital care, eg, foot clinic, vascular clinic, diabetologist, or a geriatrician.

- Each resident with diabetes should be reviewed by either their family doctor or a hospital consultant physician/geriatrician at least once a year. The review should encompass overall management and glycaemic targets in the light of changes in their physical, psychological and cognitive status.

- Patients with diabetes in residential and nursing homes should be included in any local audit of diabetic care. This should ideally include registering all diabetic residents on district diabetes registers (and on general practitioner diabetic registers, if available).

\section{Future initiatives}

Diabetes educational and training programmes for staff working in residential and nursing homes need to be established in every health district. Topics to be covered include screening for and prevention of complications of diabetes, principles of treatment, including management of hypoglycaemia, treatment of diabetic emergencies, care of the ill diabetic resident, foot infections, dietary needs of residents with diabetes, and home blood and urine glucose monitoring.

- Standards of diabetes care for patients within institutions need to be established. This will require close co-operation between primary care physicians (general practitioners) and hospital consultants (geriatricians with a special interest in diabetes; diabetologists) and input from other health professionals involved in the care of these patients. This may have to be established at a national level first (eg, the British Diabetic Association), although local initiatives through local diabetes service advisory groups should be encouraged.

- Diabetes nurse specialists for the elderly should be employed to visit all nursing and residential homes on a regular basis to review management of diabetic residents and to assist in the development of the educational and training programmes for care staff. In this way, they can also regularly update a local diabetes register to include all persons with diabetes residing in institutional care.

\section{Further reading}

1 British Diabetic Association. Diabetes care today. A guide for residential and nursing home managers and staff. 10 Queen Anne Street, London W1M OBD, UK, 1995.

2 Turnbull CJ, Sinclair AJ. Modern perspectives and recent advances. In: Finucane $P$, Sinclair AJ, eds. Diabetes in old age. London: John Wiley \& Sons Ltd, 1995.

3 Gadsby R. Care of people with diabetes who are housebound or in nursing and residential homes. Diab Gen Pract 1994; 4(3): 30-1.

4 Funnel MM, Herman WH. Diabetes care policies and practices in Michigan nursing homes. Diabetes Care 1995; 18: 862-6.

5 Sinclair AJ, Allard I, Bayer AJ. Observations of diabetes care in long-term institutional settings with measures of cognitive function and depenwith measures of cognitive function and
dency. Diabetes Care 1997; 20: 778-84.

6 Benbow SJ, Walsh A, Gill GV. Diabetes in institutionalized elderly people: a forgotten population? BMF 1997; 314: 1868-9. 